\title{
BMJ Open Protocol for a pilot single-centre, parallel-arm, randomised controlled trial of dietary inulin to improve gut health in solid organ transplantation: the DIGEST study
}

\author{
Julian Singer (10 , ${ }^{1,2}$ Yan Jun Li, ${ }^{1,2}$ Tracey Ying, ${ }^{1,2}$ Leyla J Aouad, ${ }^{1,2}$ \\ David M Gracey, ${ }^{1,2}$ Kate Wyburn, ${ }^{1,2}$ Laurence Macia, ${ }^{3,4}$ Huiling Wu, ${ }^{1,2}$ \\ Steven J Chadban ${ }^{1,2}$
}

To cite: Singer J, Li YJ, Ying T, et al. Protocol for a pilot singlecentre, parallel-arm, randomised controlled trial of dietary inulin to improve gut health in solid organ transplantation: the DIGEST study. BMJ Open 2021;11:e049184. doi:10.1136/ bmjopen-2021-049184

- Prepublication history for this paper is available online. To view these files, please visit the journal online (http://dx.doi. org/10.1136/bmjopen-2021049184).

Received 18 January 2021 Revised 16 March 2021 Accepted 24 March 2021

\section{Check for updates}

(c) Author(s) (or their employer(s)) 2021. Re-use permitted under CC BY-NC. No commercial re-use. See rights and permissions. Published by BMJ.

For numbered affiliations see end of article.

\section{Correspondence to}

Professor Steven J Chadban; steve.chadban@health.nsw. gov.au

\section{ABSTRACT}

Introduction Kidney transplantation remains the best treatment for end-stage kidney disease, however the requirement for indefinite immunosuppression increases the risk of cardiovascular disease, cancer and infection, leading to a reduction in long-term patient and graft survival. The gut microbiome is a critical determinant of health and modulates host immunity and metabolism through a number of recognised pathways, including through the production of immunomodulatory short-chain fatty acids (SCFA). Dietary supplementation with nondigestible fibre can augment the microbial production of SCFA and lead to favourable immune and metabolic outcomes, although this has yet to be shown in human kidney transplant recipients.

Methods and analysis Dietary inulin for gut health in solid-organ transplantation (DIGEST) is a single-centre, unblinded, pilot parallel-arm randomised controlled trial designed to assess the feasibility and adherence of dietary inulin, a naturally occurring dietary fibre, in the early post-transplant period in kidney transplant recipients. Participants will be randomised at day 28 post-transplant to a 4-week period of dietary inulin (10-20 g/day) in addition to standard care, or standard care alone, and followed-up until week 12 post-transplant.

The primary outcomes of the study are: (i) the feasibility of participant recruitment, randomisation and retention; (ii) adherence to the intervention (inulin) and (iii) the tolerability of inulin determined by changes in gastrointestinal symptoms as scored on the Gastrointestinal Symptom Rating Scale.

Secondary outcomes include: (1) glycaemic variability determined by continuous glucose monitoring; (2) abundance of SCFA-producing microbiota, as determined by 16 s rRNA sequencing of the faecal metagenome; (3) serum SCFA concentrations; (4) peripheral blood immune cell populations; (5) recipient inflammatory and metabolic profiles and (6) the incidence of biopsy-proven acute rejection and kidney function determined by estimated glomerular filtration rate.

Ethics and dissemination All study visits, clinical and laboratory assessments will be integrated into usual

\section{Strengths and limitations of this study}

- Dietary inulin for gut health in solid-organ transplantation (DIGEST) uses a randomised controlled design to assess the pleotropic effects of dietary inulin in the early period following kidney transplantation.

- DIGEST employs a pragmatic approach to trial design with all study visits, clinical and laboratory assessments conducted in parallel with usual posttransplant care, creating no additional healthcare encounters for participants.

- Four-day food diaries will capture dietary intake at multiple timepoints to assess for intraindividual and interindividual variation in habitual diet and fibre intake.

- In this open-label study, a glass of water without an added placebo is used as the comparator arm due to limitations in obtaining a placebo with inert actions on both the microbiome and glycaemia.

- As a single-centre study, the results may not be generalisable to all populations.

post-transplant care, creating no additional healthcare encounters or procedures. The risks associated with this study are minor. Inulin has been shown to be well tolerated across a variety of cohorts, with the occurrence of shortterm adverse gastrointestinal symptoms self-limiting. However, with gastrointestinal adverse events common following kidney transplantation, the tolerability of inulin in this cohort remains unknown. The results of DIGEST will be published in peer-reviewed journals and presented at academic conferences. This study has been approved by the Sydney Local Health District's Ethics Committee (Royal Prince Alfred Hospital Zone).

Trial registration number ACTRN12620000623998.

\section{INTRODUCTION}

Kidney transplantation is the treatment of choice for the majority of patients with end-stage kidney disease. Compared with 
enduring dialysis, kidney transplantation delivers superior survival and quality of life at a reduced financial cost. ${ }^{1-3}$ However, in the absence of long-term immunosuppression, immune-mediated rejection of the transplant invariably occurs. ${ }^{4}$ Modern immunosuppressive regimes, while effective at reducing the development of acute rejection, do so at considerable expense to both the patient and the allograft. For the majority of kidney transplant recipients (KTRs), eventual loss of a kidney transplant occurs through either death with a functioning graft, or through chronic alloimmune-mediated injury. ${ }^{5}$ The most common causes of death post-transplant (cardiovascular disease, cancer and infection) are all promoted by immunosuppression. ${ }^{6}$ Furthermore, the mainstay of current treatment regimes, calcineurin inhibitors, paradoxically contribute to premature graft loss through the development of chronic allograft nephropathy. ${ }^{78}$ Innovative strategies are hence needed to reduce the burden of current immunosuppression and maintain the balance between therapeutic efficacy and drug toxicity.

The gut microbiome is a critical determinant of human health and modulates host immunity and metabolism through a number of recognised pathways. ${ }^{9}$ In transplantation, the gut microbiome offers a novel pathway to modify maladaptive immune and metabolic responses driven by alloantigen exposure and immunosuppression. Microbiota-derived metabolites, and short-chain fatty acids (SCFA) in particular, are emerging as key mediators of the gut microbiomes influence over organ systems, including the immune system. ${ }^{10}$ Formed by the fermentation of non-digestible dietary fibre by specific colonic bacteria, SCFAs may exert local effects on gut mucosa or act systemically, following systemic absorption, via engagement of specific G-protein coupled receptors or by inhibiting histone deacetylases and altering epigenetic regulation of gene expression. ${ }^{11}{ }^{12}$ In animal studies, SCFAs or their precursors (dietary fibre) have been shown to attenuate kidney ischaemia-reperfusion injury, ${ }^{13}$ and retard the development of allograft rejection, ${ }^{14}$ as we have recently shown in a kidney allograft model. ${ }^{15}$ Furthermore, post-transplant hyperglycaemia caused by the commonly used immunosuppressive agent, tacrolimus, has been linked to a reduction in intestinal SCFAproducing bacteria, with SCFA supplementation sufficient to prevent or correct the dysglycaemic response. ${ }^{16}$

Kidney transplantation results in considerable disruption to the gut microbiome, although the complexity and high interindividual variation in microbial communities of recipients has thus far impaired the ability of small studies to identify universal microbial markers predictive of post-transplant events. ${ }^{17-19}$ However, the abundance of SCFA-producing bacteria in recipients of allogeneic haematopoietic stem cell transplants has been associated with resistance against respiratory viral infection, ${ }^{20}$ suggesting a further role for SCFA in promoting protective immunity post-transplant.

Taken together, a growing body of evidence suggests that dietary supplementation to increase the presence of
SCFA-producing bacteria may promote transplant tolerance, and protect against infective complications and reduce one of the greatest contributors to post-transplant morbidity and mortality in dysglycaemia.

Inulin is a naturally occurring non-digestible dietary fibre composed of fructo-oligosaccharide (FOS) polymers. ${ }^{21}$ In cohorts of healthy and overweight adults, dietary inulin has been shown to effectively promote the growth of SCFA-producing bacteria and increase serum SCFA concentrations, ${ }^{22-25}$ leading to an improvement in metabolic parameters, including insulin resistance and glycaemic control. ${ }^{26-28}$ Dose regimens of inulin and inulin-type fructans in interventional studies have varied widely, ${ }^{26}$ however a fixed dose of $20 \mathrm{~g}$ /day is sufficient to generate changes in gut microbiota composition while limiting untoward gastrointestinal effects. ${ }^{29}$

While dietary prebiotics have been shown to promote the growth of SCFA-producing bacteria in healthy and dysglycaemic cohorts, no studies have examined whether the gut microbiota are similarly amenable to intervention in the post-transplant period. Given the high incidence of dysglycaemia and glucose variability in the post-transplant cohort, ${ }^{30} 31$ the potential to derive a meaningful advantage from even small improvements in glucose metabolism is significant.

This pilot, randomised controlled trial will assess the feasibility and adherence to dietary inulin supplementation, and explore the effect of inulin on gut bacterial communities and glycaemic variability in the early posttransplant period. This trial aims to provide data to inform the feasibility, design and viability of larger clinical trials to assess the efficacy of dietary prebiotics in improving outcomes for KTRs.

\section{METHODS AND ANALYSIS \\ Study design}

The Dietary Inulin for Gut Health in Solid-organ Transplantation (DIGEST) study is a single-centre, unblinded, parallel-arm randomised controlled trial of 40 adult KTRs. The trial will screen patients for enrolment from post-transplant day 14 and randomise participants in a 1:1 ratio at post-transplant day 28 to a 4-week period of either (a) supplementation with dietary inulin in addition to standard post-transplant care or (b) standard posttransplant care alone. Study participants will be followed until post-transplant week 12. An outline of the study design is illustrated in figure 1.

\section{Study aim and hypothesis}

The primary aims of DIGEST are to determine the feasibility of recruitment, randomisation and retention; and the adherence to and tolerance of inulin supplementation in the early post-transplant period. The secondary outcomes seek to evaluate the effect of inulin supplementation on the relative abundance of SCFA-producing gut bacteria, glycaemic variability, metabolic profiles, immune cell populations and graft outcomes. These 


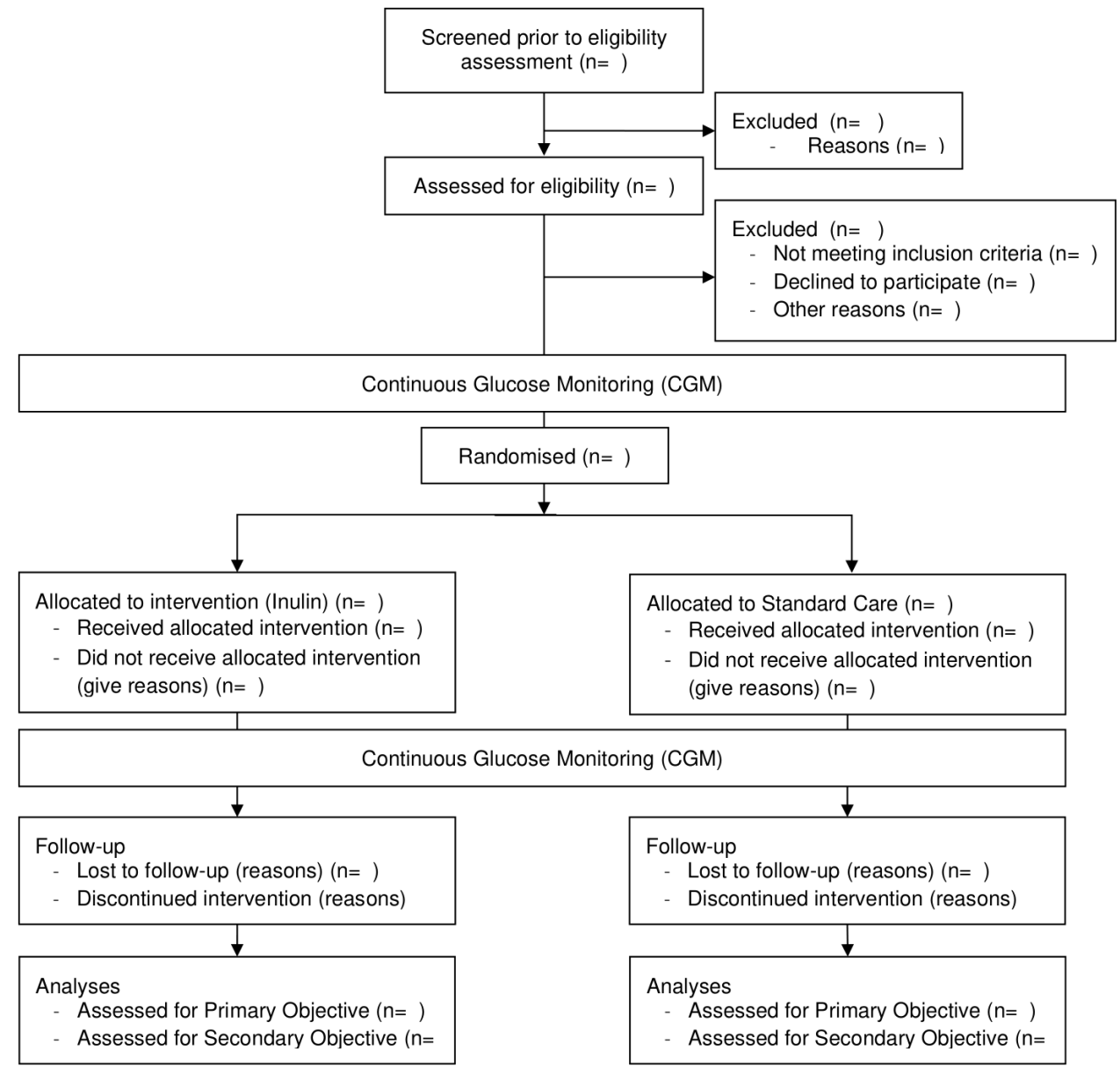

Figure 1 Study flow chart for the Dietary inulin for gut health in solid-organ transplantation pilot trial, adapted from the Consolidated Standards of Reporting Trials extension to randomised pilot and feasibility trials.

exploratory outcomes will assess data collection and outcome measures, and will be used to inform the design of a definitive trial.

We hypothesise that dietary prebiotic inulin is feasible, safe and well tolerated in the early post-transplant period. In addition, we seek to show that inulin will favourably enhance the relative abundance of SCFA-producing gut bacteria and reduce the range of glycaemic variability post-transplant.

\section{Target population and eligibility criteria}

DIGEST will enrol adult (18 years or older) recipients of kidney transplants performed at Royal Prince Alfred Hospital, a quarternary-care hospital in Sydney, Australia from 16 June 2020 until the recruitment target is met.

\section{Inclusion criteria}

The inclusion and exclusion criteria are shown in box 1 . DIGEST will recruit both diabetic and non-diabetic KTRs from deceased or living donors who are willing and able to provide written informed consent and are able to comply with all trial and follow-up requirements for the duration of the study.

\section{Exclusion criteria}

The complete exclusion criteria are shown in box 1 . Conditions with the potential to confound the interpretation of the intervention (inulin) on outcome measures will be excluded. These include: (1) KTRs with significant gastrointestinal disorders (such as coeliac disease or inflammatory bowel disease) or a prior colonic resection; (2) KTRs at higher immune risk who receive T-cell depleting agents and those with acute rejection who are subsequently exposed to greater levels of immunosuppression and (3) KTRs with delayed graft function requiring dialysis beyond post-transplant day 14 .

\section{Recruitment and randomisation}

Potential participants will be identified for inclusion in the study by clinical staff or a member of the research team, either at the time of their initial hospital stay following kidney transplantation, or during their regular attendance at the acute transplant clinic following hospital discharge. All potentially eligible patients will be screened for enrolment, provided with a study information sheet, and if agreeable, receive information regarding the study from a research team member. Prior to performing any study-specific procedure, a signed consent form will be obtained for each subject. For subjects without capacity 


\section{Box 1 Inclusion and exclusion criteria}

\section{Inclusion criteria}

- Recipients of a kidney transplant from a living or deceased donor.

- Individuals aged $\geq 18$ years who are able to give informed consent, and a willingness to participate and comply with the study requirements.

- Patients who receive a kidney from an $\mathrm{ABO}$ blood group incompatible donor, or as part of the Paired Kidney Exchange Programme.

\section{Exclusion criteria}

- Individuals diagnosed with significant gastrointestinal diseases, for example, inflammatory bowel disease, coeliac disease.

- Patients who receive antithymocyte globulin as induction or treatment for rejection.

- Acute rejection in the first 4 weeks post-transplant.

- Delayed graft function requiring dialysis persisting beyond week 2 post-transplant.

- Presence of gastrointestinal tract output stoma.

- Current enrolment in another intervention or investigational drug trial.

- Recipients of multiorgan transplants.

- Known food intolerance, allergy or sensitivity to inulin or dietary fibre.

- Inability or unwillingness of individual or legal guardian to give written informed consent.

to consent, a signed consent form will be obtained from their legal guardian. A study investigator will conduct the informed consent discussion to ensure that the patient and/or legal representative comprehends the information provided, and that all queries or concerns are addressed. Consent will be voluntary and free from coercion.

Randomisation will occur through computer-generated permuted block randomisation using a block size of 6 , with participants assigned in a 1:1 ratio to either (a) supplementation with dietary inulin dissolved in 200 $\mathrm{mL}$ water two times per day in addition to standard posttransplant care or (b) $200 \mathrm{~mL}$ water two times per day in addition to standard post-transplant care. Randomisation will be stratified by sex and diabetic status at the time of study enrolment to maintain the balance of confounders between the two study arms. The randomisation schedule will be generated by the "blockrand" ${ }^{32}$ package for $\mathrm{R}$, and administered through a central web-based randomisation module contained within the REDCap data management platform, ${ }^{33}$ ensuring concealment of the allocation sequence from investigators. DIGEST is an open-label trial, with participants and investigators unblinded to study-arm allocation once randomisation has occurred. Following completion of all data collection, the study-arms will be relabelled with non-identifying terms by a third party to blind the data analysts to treatment allocation.

\section{Intervention period}

The intervention period of the DIGEST trial will run for 4 weeks, commencing at day 28 post-transplant.

\section{Intervention group}

Participants randomised to the intervention arm will receive inulin $10 \mathrm{~g}$ each morning for the first 7 days, increasing to $10 \mathrm{~g}$ morning and night for the remaining 21 days of the intervention period. A run-in period of inulin $10 \mathrm{~g}$ once daily followed by dose escalation to $10 \mathrm{~g}$ two times per day after 1 week has been used to minimise the development of early dose-related unacceptable adverse gastrointestinal effects. Inulin will be provided in powdered form and contained within individual $10 \mathrm{~g}$ sachets. The contents of each sachet will be self-administered by the study participant by dissolving in approximately $200 \mathrm{~mL}$ of water prior to consuming. The study supplement will be acquired from MYPROTEIN in its commercially available form.

Inulin is a naturally occurring FOS composed of heterogenous polymers of 2-60 d-fructose units with each ending in a terminal glucosyl unit. It may be produced synthetically through enzymatic action on sucrose or extracted from a natural source, that is, chicory root. It is available as a retail food additive or dietary supplement in a powdered form and is readily soluble in water with a neutral unflavoured taste.

Participants randomised to the intervention group will continue with standard post-transplant care as documented below.

\section{Monitoring}

Subjects who experience persistent mild adverse effects (bowel discomfort, bloating, flatulence) will be instructed to reduce the dose to $10 \mathrm{~g} /$ day. Subjects who are unable to continue with the supplement will discontinue the study intervention and continue with all study visits and assessments. All protocol deviations and adverse events will be recorded.

\section{Standard care group}

During the intervention period, participants in the standard care group are asked to consume one glass of water (approximately $200 \mathrm{~mL}$ ) each morning for the first 7 days, increasing to morning and night for the following 21 days.

Participants in both arms of the study will receive standard post-transplant care as per the local practice guidelines. This includes standard immunosuppression for the prevention of immune-mediated rejection (calcineurin inhibitor, mycophenolate and glucocorticoids), and supportive treatments to manage medical risks posttransplant such as antimicrobial prophylaxis for Pneumocystis jirovecii, cytomegalovirus and Candida, in addition to antihypertensive and hypoglycaemic medications at the direction of their transplant physician. All patients receive medication education through a pharmacist, and nutritional assessment and advice by a renal dietitian either during their inpatient hospital stay or as part of their regular follow-up in the acute transplant clinic. Enroled participants will be instructed to continue with 


\begin{tabular}{|c|c|c|c|c|c|c|c|c|c|}
\hline & \multicolumn{9}{|c|}{ STUDY PERIOD } \\
\hline \multirow[b]{2}{*}{$\begin{array}{r}\text { TIMEPOINT } \\
\text { (time in weeks) }\end{array}$} & Enrolment & & Allocation & \multicolumn{5}{|c|}{ Post-allocation } & \multirow{2}{*}{$\begin{array}{c}\text { Close-out } \\
8 \pm 1\end{array}$} \\
\hline & -2 & -1 & 0 & 1 & 2 & 3 & 4 & 5 & \\
\hline \multicolumn{10}{|l|}{ ENROLMENT: } \\
\hline Eligibility screen & $x$ & & & & & & & & \\
\hline Informed consent & $X$ & & & & & & & & \\
\hline Allocation & & & $x$ & & & & & & \\
\hline \multicolumn{10}{|l|}{ INTERVENTIONS: } \\
\hline \multicolumn{10}{|l|}{ Inulin + Standard Care } \\
\hline \multicolumn{10}{|l|}{ Standard Care } \\
\hline \multicolumn{10}{|l|}{ ASSESSMENTS: } \\
\hline Demographics & $x$ & & & & & & & & \\
\hline Transplant characteristics & $x$ & & & & & & & & \\
\hline CGM & & $\vdash$ & & $\rightarrow$ & & & $\vdash$ & $\rightarrow$ & \\
\hline Height & $x$ & & & & & & & & \\
\hline Weight & 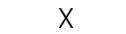 & $\mathrm{X}$ & $x$ & $x$ & & & $x$ & $x$ & $x$ \\
\hline Blood pressure & 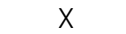 & $\mathrm{X}$ & $x$ & $X$ & & & $x$ & $x$ & 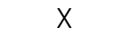 \\
\hline Current Medications & $x$ & $x$ & $\mathrm{X}$ & $x$ & & & $x$ & $x$ & $x$ \\
\hline GSRS & & & $x$ & $x$ & & & $x$ & & $x$ \\
\hline $\begin{array}{r}\text { Haematological and } \\
\text { biochemical tests }\end{array}$ & & & $x$ & & & & $x$ & & $x$ \\
\hline Stool collection & & & $x$ & & & & $x$ & & $x$ \\
\hline 4-day food diary & & & $x$ & & & & $x$ & & $x$ \\
\hline $75 \mathrm{~g}$ OGTT* & & & & & & & & & $x$ \\
\hline Protocol biopsy ${ }^{\dagger}$ & & & & & & & & & $X$ \\
\hline Adherence assessment & & & & $x$ & $x$ & $x$ & $x$ & & \\
\hline $\begin{array}{r}\text { Adverse event } \\
\text { assessment }\end{array}$ & & & $X$ & $X$ & $x$ & $x$ & $x$ & $x$ & X \\
\hline
\end{tabular}

Figure 2 Schematic diagram demonstrating the schedule of enrolment, intervention, assessments and visits for study participants. *For participants without diabetes. †At the discretion of the participants treating team. CGM, continuous glucose monitoring; GSRS, gastrointestinal symptoms rating scale; OGTT, oral glucose tolerance test.

their normal diet and to not increase the consumption of fibre-rich foods.

\section{Post-randomisation visits and assessment}

DIGEST employs a pragmatic approach to trial design with all study visits, clinical and laboratory assessments conducted in parallel with usual post-transplant care. For study participants, this creates no additional hospital encounters, exposes participants to no further medical procedures (including venepuncture), reduces the time commitment required for participation and will aid in the follow-up and retention of enrolled participants.
A schematic diagram of the schedule of enrolment, interventions and assessments is provided in figure 2 .

\section{Primary outcomes}

The DIGEST study has been developed to collect and report outcome data in accordance with the Consolidated Standards of Reporting Trials extension to randomised pilot and feasibility trials (figure 1 ). ${ }^{34}$

Feasibility of recruitment and retention

The feasibility of patient recruitment to the DIGEST trial will be determined by the percentage of patients 
screened who proceed to randomisation. The feasibility of retaining patients throughout the study will be assessed by the percentage of randomised patients who complete all study visits, and the feasibility of implementing the study protocol by the percentage of randomised patients who complete all outcome measures. The feasibility of recruitment, retention and implementation will be determined at the completion of the final study visit by the last enrolled participant.

\section{Adherence and tolerability of inulin}

The adherence to inulin will be determined by the number of subjects randomised to the intervention arm who consume at least $10 \mathrm{~g}$ of inulin each day for $\geq 80 \%$ of the intervention period. To enhance the validity of data, multiple methods will be used to assess intervention inherence. Following allocation, participants will be questioned on the frequency of missed, skipped or omitted doses, and the number of consumed and unopened inulin sachets will be counted at each study visit. Participants will be reminded at each study visit of the importance of adherence to the study intervention and questioned for the development of adverse events. All reasons for nonadherence will be documented.

The tolerability of inulin supplementation determined by the development of adverse gastrointestinal effects will be assessed using the Gastrointestinal Symptom Rating Scale (GSRS). The GSRS is a validated, self-administered questionnaire, which assesses the severity of gastrointestinal symptoms using a 7-point Likert scale across five domains: indigestion, diarrhoea, constipation, abdominal pain and reflux. It has previously been validated in the renal transplant population. ${ }^{35}$ The GSRS will be administered to participants in both trial arms just prior to, and 1 week following the commencement of the intervention period, at the end of the intervention period and at post-transplant week 12. Patients who require either dose reduction or cessation of inulin due to adverse gastrointestinal effects will be recorded.

\section{Safety}

All adverse events will be recorded using an adaptation of the National Institute of Health's Common Terminology Criteria for Adverse Events by a study team member. All study participants will be screened for the occurrence of adverse events at each study visit by a member of the research team, and during their regular assessment by a transplant physician in the acute transplant clinic. All serious adverse events will be reported to the study sponsor within 24 hours of the study team becoming aware of the event.

\section{Secondary outcomes}

Secondary outcomes for the DIGEST study are exploratory in nature and will be performed to examine the feasibility of data collection and biospecimen analysis, and to inform the viability of the study protocol for a definitive trial.

\section{Continuous glucose monitoring}

Glycaemic control and variability will be measured through continuous glucose monitoring (CGM) conducted over two 14-day periods using a Libre Freestyle sensor. CGM sensors will be placed on all study participants at 1 week prior to the commencement, and 1 week prior to the cessation of the intervention period. Data generated from CGM will be analysed and reported in line with current guidelines for CGM reporting. ${ }^{37}$

\section{Clinical and laboratory measurements}

Study participants will have clinical and laboratory outcomes assessed at three time points during the study: (i) prior to the commencement of the intervention period; (ii) within the last week of the intervention period and (iii) at the final study visit occurring at post-transplant week 12. These measures include weight and body mass index, blood pressure, serum creatinine, lipid profile, fasting glucose and insulin levels and blood short-chain fatty acid levels. Plasma and peripheral blood mononuclear cells isolated from whole blood will be aliquoted and stored for biochemical analysis and immunophenotyping by cytometry, respectively. Patients without diabetes will be asked to undergo a $75 \mathrm{~g}$ oral glucose tolerance test following week 10 post-transplant to assess for the presence of impaired glucose tolerance or the occult occurrence of new-onset diabetes after transplant. Indication and 12-week protocol biopsies of the kidney transplant will be performed at the discretion of the participant's treating clinicians.

\section{Faecal microbiota}

16s rRNA sequencing of the faecal metagenome isolated from stool samples collected at the above timepoints will be used to determine the diversity, composition and relative abundance of bacteria within the gut microbiome. Faecal specimens are collected using an all-in-one system (OMNIgene GUT OM-200, DNA Genotek, Canada) for easy self-collection by the participant in their home environment and point-of-collection stabilisation of faecal DNA at room temperature, until it can be returned to a study team member. An assessment of habitual diet will be determined by 4-day food diaries recorded at the time of stool sample collection and analysed via FoodWorks.

\section{Patient and public involvement}

While the impetus for this pilot study is derived principally from our preclinical research, informal discussions with KTRs revealed significant interest in the relationship between diet, kidney health and metabolic disease following transplantation, and a willingness to participate in research. Prior to finalisation of the study protocol, we consulted three recent KTRs regarding the design, implementation and data collection methods for this trial. Their feedback resulted in modifications to the participant information sheet and adaptation of the study visits and data collection tools to more adequately respond to the needs of patients in the early 
post-transplant period, and to reduce the burden of time required to participate in the research.

\section{Sample size calculation}

This pilot study aims to determine the tolerability and feasibility of inulin supplementation in the early post-transplant period, with exploratory outcomes that may provide initial data for primary outcome measures in order to calculate sample size for a larger trial.

The use of metrics derived from CGM remains a novel outcome measure. We have previously demonstrated in a cohort of 28 renal transplant recipients significant variations in glucose homeostasis with the use of CGM, identifying a role for CGM in predicting patients who will go on to develop post-transplant diabetes. ${ }^{30}$ Interventional studies assessing the impact of dietary fibre on glucose metabolism have not reported CGM outcomes, while commonly reported measures such as haemoglobin Alc have decreased diagnostic utility in the early post-transplant period. ${ }^{38}$

Using reported data of available glycaemic metrics, a meta-analysis examining the metabolic benefits of dietary prebiotics in non-transplant cohorts found that dietary prebiotics (fibre) improved postprandial glucose levels with a standardised mean difference of $-0.79 \mathrm{mmol} / \mathrm{L}(95 \%$ CI -1.41 to -0.12$).{ }^{27}$ For a main trial designed to detect a standardised effect size of 0.8 at $90 \%$ power and two-sided $5 \%$ significance, pilot trial sample sizes of 10 per treatment arm have been recommended. ${ }^{39}$ We therefore aim to recruit 20 subjects per treatment arm, allowing for both drop-out and a margin of error in effect size.

\section{Data analysis plan}

The intention-to-treat principle for all outcomes will be applied to the final analyses with patients assessed according to their trial arm allocation; however, we will also report perprotocol results. ${ }^{40}$ The per-protocol analyses will exclude patients from the treatment arm who failed to adhere or tolerate inulin supplementation, or consumed $<80 \%$ of the prescribed supplement.

The following analyses will be performed for the primary outcome: (1) recruitment and retention of trial participants will be reported as the percentage of patients screened who proceed to randomisation, and the percentage of randomised patients who completed all study procedures and follow-up requirements, respectively; (2) adherence to inulin will be reported as the percentage of participants randomised to the intervention arm who adhere to inulin supplementation for the full prescribed period and (3) the tolerability of inulin determined by longitudinal changes in GSRS scores analysed using a generalised linear mixed model with study arm, time, sex, age, immunosuppression type, antibiotic use in the prior week (yes or no) and the interaction between study arm and time as the main fixed effects. The random effect of subject will be used to account for the repeated interdependent observations from each study subject. Data missing at random will be handled in a mixed model using maximum likelihood estimates. If $>10 \%$ of primary outcome data is determined to be missing not at random, a best-worst and worst-best case sensitivity analyses will be performed.

Baseline characteristics of participants and recorded outputs will be expressed as means \pm SD for normally distributed data or the median $\pm \mathrm{IQR}$ for non-normally distributed data, and as frequencies for categorical variables. Differences in continuous variables between the study arms will be assessed using Student's t-test for normally distributed data, or by the non-parametric Wilcoxon signed rank test for non-normally distributed data. Categorical variables will be compared using the $\chi^{2}$ and Fisher's exact test as appropriate. Exploratory outcome measures will be analysed by univariate and multivariate methods with adjustments for multiple comparisons where required. A predefined subgroup analysis by diabetic status will be performed for the exploratory outcomes. A two-sided significance level of $5 \%$ will be used for all analyses.

\section{Data management and access}

A Research Data Management Plan (RDMP) has been created and approved under local ethics governance. All study data are entered directly onto study-specific electronic data capture forms coordinated through a secure web-based data management tool called REDCap. ${ }^{33}$ The stored data are maintained on protected servers with the Sydney Local Health District data centre, ensuring provision of back-up, privacy and confidentiality requirements. Access to the secure REDCap DIGEST database is available only to research team members. REDCap requires individual user authentication, ensures data logging and uses Secure Sockets Layer encryption, allowing data integrity and monitoring to be upheld as per the RDMP. All biobanked laboratory specimens are labelled with a unique coded identifier to maintain participant confidentiality.

\section{ETHICS AND DISSEMINATION}

The safety and tolerability of inulin has been widely studied across a number of cohorts where it has been shown to be safe and well tolerated in doses exceeding $20 \mathrm{~g} /$ day. ${ }^{41-43}$ The possible benefits to participants include any potential benefit derived from the study intervention, additionally participants with prior or new-onset diabetes after transplant may derive benefit from the periods of CGM with the reduced requirement for burdensome finger-prick testing of capillary blood glucose.

The results of DIGEST are anticipated to be published in peer-reviewed scientific journals and presented at academic meetings. We anticipate the findings will be of interest across a broad field of transplant clinicians, nephrologists, dietitians and consumers. Identifiable data will not be publicly released and deidentified data may be made available on reasonable request from interested investigators, with the exception of faecal metagenome sequencing reads which will be uploaded to the European Nucleotide Archive (EMBL-EBI). Trial data will be held by the Sydney Local Health District and the University of Sydney for a minimum period of 15 years. 
This study has been approved by the Sydney Local Health District's Ethics Committee (Royal Prince Alfred Hospital Zone) under project ID 2019/PID14472.

\section{DISCUSSION}

Assessing the feasibility, adherence and tolerance of dietary fibre supplementation by KTRs is the first step in determining whether prebiotic fibre may have a role as an adjunctive therapy to improve outcomes for KTRs. The information derived from this pilot study will provide estimates of the eligibility, recruitment and retention rates that will inform the design, size and feasibility of future studies. Larger scale trials will be required to more conclusively determine whether dietary supplementation can alter the gut microbiome post-transplant, and ultimately improve transplant outcomes.

Modifying the gut microbiota by dietary supplementation is a novel and promising strategy to promote health outcomes that has garnered much public interest. ${ }^{445}$ Indeed, consultation with our own patients suggests a willingness to engage in this area of research. Taken with the knowledge that dietary supplement use is common in Australia, with 47\% of women and $34 \%$ of men consuming supplements regularly, ${ }^{46}$ we believe there will be significant interest in this study by potential participants, in addition to the scientific community.

Prebiotic dietary fibres are a key nutritional source for SCFA-producing gut bacteria (eg, bifidobacteria and lactobacillus) and promote their selective growth. ${ }^{22}{ }^{23}$ As the prototypical prebiotic, inulin has been commonly used as an investigational product and has established a reputable body of literature demonstrating both safety and efficacy. ${ }^{42} 434748$ Together with inulin's wide availability and low cost, it forms an ideal investigational supplement for use in the posttransplant period. However, with gastrointestinal adverse events common in KTRs, ${ }^{49}$ the adherence and tolerability of inulin supplementation in this cohort remains unknown and requires investigation.

The early post-transplant period presents numerous challenges for KTRs that must be acknowledged. New medications with frequent dosing changes, adaptation to new healthcare settings, intercurrent illness and an exposure to an excess of information may all reduce KTRs willingness to participate in clinical trials. ${ }^{50}$ In this regard, there are a number of factors that we believe will enhance interest and participation in this trial. The DIGEST trial creates no additional healthcare encounters for participants, which is of critical importance during this period of increased risk with COVID-19. Furthermore, the short follow-up period in this pilot trial is intended to enhance participation during the 'acute phase' of post-transplant care, prior to a patient's discharge from the primary transplant centre, often to distant locations.

DIGEST is not without limitations. The unique physical and metabolic properties of inulin (soluble, non-digestible, fermentable fibre) preclude the attainment of a suitable placebo that will maintain an inert effect on both participant glycaemia and the gut microbiome, while preserving participant and investigator blinding to treatment allocation. Previous controls in inulin trials have frequently included maltodextrins, ${ }^{51}{ }^{52}$ which have a high glycaemic index, or non-fermentable microcrystalline cellulose ${ }^{53} 54$ which is insoluble in water. Furthermore, we acknowledge that commencing a dietary supplement to establish a favourable microbiome prior to transplant would increase the likelihood of attaining a microbiota-derived benefit. However, this would limit participation to a smaller cohort of livingdonor transplant recipients or render wait-listed patients to long-term dietary supplementation.

Ultimately, the DIGEST pilot study will provide important information in a novel area of transplant medicine and inform the direction of future interventional trials.

\section{Author affiliations}

${ }^{1}$ Kidney Node Laboratory, Charles Perkins Centre, The University of Sydney, Sydney, New South Wales, Australia

2Department of Renal Medicine, Royal Prince Alfred Hospital, Sydney, New South Wales, Australia

${ }^{3}$ Nutritional Immunometabolism Node, Charles Perkins Centre, The University of Sydney, Sydney, New South Wales, Australia

${ }^{4}$ School of Medical Sciences, Faculty of Medicine and Health, The University of Sydney, Sydney, New South Wales, Australia

Contributors The study was conceived and designed by JS, LM, HW and SJC. JS wrote the first draft of the DIGEST protocol and prepared the manuscript. YJL, TY, LJA, DMG, KW, LM, HW and SJC participated in critical review of the study protocol, and revisions to the manuscript. All authors contributed significant intellectual content to the protocol and approved the final manuscript.

Funding The authors have not declared a specific grant for this research from any funding agency in the public, commercial or not-for-profit sectors.

\section{Competing interests None declared.}

Patient and public involvement Patients and/or the public were involved in the design, or conduct, or reporting, or dissemination plans of this research. Refer to the Methods section for further details.

Patient consent for publication Not required.

Provenance and peer review Not commissioned; externally peer reviewed.

Open access This is an open access article distributed in accordance with the Creative Commons Attribution Non Commercial (CC BY-NC 4.0) license, which permits others to distribute, remix, adapt, build upon this work non-commercially, and license their derivative works on different terms, provided the original work is properly cited, appropriate credit is given, any changes made indicated, and the use is non-commercial. See: http://creativecommons.org/licenses/by-nc/4.0/.

ORCID iD

Julian Singer http://orcid.org/0000-0002-1855-3581

\section{REFERENCES}

1 Tonelli M, Wiebe N, Knoll G, et al. Systematic review: kidney transplantation compared with dialysis in clinically relevant outcomes. Am J Transplant 2011;11:2093-109.

2 Wolfe RA, Ashby VB, Milford EL, et al. Comparison of mortality in all patients on dialysis, patients on dialysis awaiting transplantation, and recipients of a first cadaveric transplant. $N$ Engl J Med 1999;341:1725-30.

3 Axelrod DA, Schnitzler MA, Xiao H, et al. An economic assessment of contemporary kidney transplant practice. Am J Transplant 2018;18:1168-76.

4 Nankivell BJ, Alexander SI. Rejection of the kidney allograft. N Engl J Med 2010;363:1451-62.

5 Pascual M, Theruvath T, Kawai T, et al. Strategies to improve long-term outcomes after renal transplantation. N Engl J Med 2002;346:580-90. 
6 Ying T, Shi B, Kelly PJ, et al. Death after kidney transplantation: an analysis by era and time post-transplant. J Am Soc Nephrol 2020;31:ASN.2020050566:2887-99.

7 Stoumpos S, Jardine AG, Mark PB. Cardiovascular morbidity and mortality after kidney transplantation. Transp/ Int 2015;28:10-21.

8 Legendre C, Canaud G, Martinez F. Factors influencing long-term outcome after kidney transplantation. Transpl Int 2014;27:19-27.

9 Clemente JC, Ursell LK, Parfrey LW, et al. The impact of the gut microbiota on human health: an integrative view. Cell 2012;148:1258-70.

10 Tan J, McKenzie C, Potamitis M, et al. The role of short-chain fatty acids in health and disease. Adv Immunol 2014;121:91-119.

11 Maslowski KM, Vieira AT, Ng A, et al. Regulation of inflammatory responses by gut microbiota and chemoattractant receptor GPR43. Nature 2009;461:1282-6.

12 Fellows R, Denizot J, Stellato C, et al. Microbiota derived short chain fatty acids promote histone crotonylation in the colon through histone deacetylases. Nat Commun 2018;9:105.

13 Andrade-Oliveira V, Amano MT, Correa-Costa M, et al. Gut bacteria products prevent AKI induced by ischemia-reperfusion. J Am Soc Nephrol 2015;26:1877-88.

14 Bromberg JS, Hittle L, Xiong Y, et al. Gut microbiota-dependent modulation of innate immunity and lymph node remodeling affects cardiac allograft outcomes. JCI Insight 2018;3:e121045.

$15 \mathrm{Wu} \mathrm{H}$, Singer J, Kwan TK, et al. Gut microbial metabolites induce donor-specific tolerance of kidney allografts through induction of T regulatory cells by short-chain fatty acids. J Am Soc Nephrol 2020;31:1445-61.

16 Jiao W, Zhang Z, Xu Y, et al. Butyric acid normalizes hyperglycemia caused by the tacrolimus-induced gut microbiota. Am J Transplant 2020;20:2413-24.

17 Fricke WF, Maddox C, Song Y, et al. Human microbiota characterization in the course of renal transplantation. Am J Transplant 2014;14:416-27.

18 Lee JR, Muthukumar T, Dadhania D, et al. Gut microbial community structure and complications after kidney transplantation: a pilot study. Transplantation 2014;98:697-705.

19 Lee JR, Magruder M, Zhang L, et al. Gut microbiota dysbiosis and diarrhea in kidney transplant recipients. Am J Transplant 2019;19:488-500.

20 Haak BW, Littmann ER, Chaubard J-L, et al. Impact of gut colonization with butyrate-producing microbiota on respiratory viral infection following allo-HCT. Blood 2018;131:blo od-2018-01-828996-86.

21 Niness KR. Inulin and Oligofructose: what are they? J Nutr 1999;129:1402S-6.

22 So D, Whelan K, Rossi M, et al. Dietary fiber intervention on gut microbiota composition in healthy adults: a systematic review and meta-analysis. Am J Clin Nutr 2018;107:965-83.

23 Bouhnik Y, Raskine L, Simoneau G, et al. The capacity of shortchain fructo-oligosaccharides to stimulate faecal bifidobacteria: a dose-response relationship study in healthy humans. Nutr $J$ 2006;5:8.

24 Calame W, Weseler AR, Viebke C, et al. Gum arabic establishes prebiotic functionality in healthy human volunteers in a dosedependent manner. Br J Nutr 2008;100:1269-75.

25 Meyer D, Stasse-Wolthuis M. The bifidogenic effect of inulin and oligofructose and its consequences for gut health. Eur J Clin Nutr 2009;63:1277-89.

26 Beserra BTS, Fernandes R, do Rosario VA, et al. A systematic review and meta-analysis of the prebiotics and synbiotics effects on glycaemia, insulin concentrations and lipid parameters in adult patients with overweight or obesity. Clin Nutr 2015;34:845-58.

27 Kellow NJ, Coughlan MT, Reid CM. Metabolic benefits of dietary prebiotics in human subjects: a systematic review of randomised controlled trials. Br J Nutr 2014;111:1147-61.

28 Wang $\mathrm{L}$, Yang $\mathrm{H}$, Huang $\mathrm{H}$, et al. Inulin-type fructans supplementation improves glycemic control for the prediabetes and type 2 diabetes populations: results from a GRADE-assessed systematic review and dose-response meta-analysis of 33 randomized controlled trials. $J$ Transl Med 2019;17:410.

29 Bruhwyler J, Carreer F, Demanet E, et al. Digestive tolerance of inulin-type fructans: a double-blind, placebo-controlled, cross-over, dose-ranging, randomized study in healthy volunteers. Int J Food Sci Nutr 2009;60:165-75.

30 Aouad LJ, Clayton P, Wyburn KR, et al. Evolution of glycemic control and variability after kidney transplant. Transplantation 2018;102:1563-8.
31 Kasiske BL, Snyder JJ, Gilbertson D, et al. Diabetes mellitus after kidney transplantation in the United States. Am J Transplant 2003;3:178-85

32 Snow G. blockrand: randomization for block random clinical trials. $\mathrm{R}$ package version 14/r13, 2019. Available: https://R-Forge.R-project. org/projects/blockrand/

33 Harris PA, Taylor R, Thielke R, et al. Research electronic data capture (REDCap)--a metadata-driven methodology and workflow process for providing translational research informatics support. J Biomed Inform 2009;42:377-81.

34 Eldridge SM, Chan CL, Campbell MJ, et al. Consort 2010 statement: extension to randomised pilot and feasibility trials. BMJ 2016;355:i5239.

35 Kleinman L, Faull R, Walker R, et al. Gastrointestinal-specific patient-reported outcome instruments differentiate between renal transplant patients with or without Gi complications. Transplant Proc 2005;37:846-9.

36 Svedlund J, Sjödin I, Dotevall G. GSRS--a clinical rating scale for gastrointestinal symptoms in patients with irritable bowel syndrome and peptic ulcer disease. Dig Dis Sci 1988;33:129-34.

37 Battelino T, Danne T, Bergenstal RM, et al. Clinical targets for continuous glucose monitoring data interpretation: recommendations from the International consensus on time in range. Diabetes Care 2019;42:1593-603.

38 Pimentel AL, Cavagnolli G, Camargo JL. Diagnostic accuracy of glycated hemoglobin for post-transplantation diabetes mellitus after kidney transplantation: systematic review and meta-analysis. Nephrol Dial Transplant 2017;32:gfw437-72.

39 Whitehead AL, Julious SA, Cooper CL, et al. Estimating the sample size for a pilot randomised trial to minimise the overall trial sample size for the external pilot and main trial for a continuous outcome variable. Stat Methods Med Res 2016;25:1057-73.

40 Schulz KF, Altman DG, Moher D, et al. Consort 2010 statement: updated guidelines for reporting parallel group randomised trials. Trials 2010;11:32

41 Carabin IG, Flamm WG. Evaluation of safety of inulin and oligofructose as dietary fiber. Regul Toxicol Pharmacol 1999;30:268-82.

42 Bonnema AL, Kolberg LW, Thomas W, et al. Gastrointestinal tolerance of chicory inulin products. J Am Diet Assoc 2010;110:865-8.

43 Joossens M, De Preter V, Ballet V, et al. Effect of oligofructoseenriched inulin (OF-IN) on bacterial composition and disease activity of patients with Crohn's disease: results from a double-blinded randomised controlled trial. Gut 2012;61:958-58.

44 Cani PD. Human gut microbiome: hopes, threats and promises. Gut 2018;67:1716-25.

45 Brody H. The gut microbiome. Nature 2020;577:S5.

46 Burnett A, Livingstone K, Woods J, et al. Dietary supplement use among Australian adults: findings from the 2011-2012 national nutrition and physical activity survey. Nutrients 2017;9:1248.

47 Holscher HD, Doligale JL, Bauer LL, et al. Gastrointestinal tolerance and utilization of Agave inulin by healthy adults. Food Funct 2014;5:1142-9.

48 Liu F, Prabhakar M, Ju J, et al. Effect of inulin-type fructans on blood lipid profile and glucose level: a systematic review and meta-analysis of randomized controlled trials. Eur J Clin Nutr 2017;71:9-20.

49 Gioco R, Corona D, Ekser B, et al. Gastrointestinal complications after kidney transplantation. World J Gastroenterol 2020;26:5797-811.

50 Gordon EJ. Rethinking how we promote and support transplantation research. Ethics Hum Res 2019;41:36-40.

51 Dehghan P, Pourghassem Gargari B, Asghari Jafar-abadi M, Gargari BP, Jafar-Abadi MA. Oligofructose-enriched inulin improves some inflammatory markers and metabolic endotoxemia in women with type 2 diabetes mellitus: a randomized controlled clinical trial. Nutrition 2014;30:418-23.

52 Pourghassem Gargari B, Dehghan P, Aliasgharzadeh A, et al. Effects of high performance inulin supplementation on glycemic control and antioxidant status in women with type 2 diabetes. Diabetes Metab J 2013;37:140-8

53 Chambers ES, Byrne CS, Morrison DJ, et al. Dietary supplementation with inulin-propionate ester or inulin improves insulin sensitivity in adults with overweight and obesity with distinct effects on the gut microbiota, plasma metabolome and systemic inflammatory responses: a randomised cross-over trial. Gut 2019;68:1430-8.

54 Guess ND, Dornhorst A, Oliver N, et al. A randomised crossover trial: the effect of inulin on glucose homeostasis in subtypes of prediabetes. Ann Nutr Metab 2016;68:26-34. 